PROCEEDINGS OF THE

AMERICAN MATHEMATICAL SOCIETY

Volume 129, Number 3, Pages 793-805

S 0002-9939(00)05743-9

Article electronically published on September 19, 2000

\title{
BLOW UP OF SOLUTIONS OF THE CAUCHY PROBLEM FOR A WAVE EQUATION WITH NONLINEAR DAMPING AND SOURCE TERMS AND POSITIVE INITIAL ENERGY
}

\author{
HOWARD A. LEVINE AND GROZDENA TODOROVA
}

(Communicated by David S. Tartakoff)

\begin{abstract}
In this paper we consider the long time behavior of solutions of the initial value problem for semi-linear wave equations of the form

$$
u_{t t}+a\left|u_{t}\right|^{m-1} u_{t}-\Delta u=b|u|^{p-1} u \quad \text { in }[0, \infty) \times R^{n} .
$$

Here $a, b>0$.

We prove that if $p>m \geq 1$, then for any $\lambda>0$ there are choices of initial data from the energy space with initial energy $\mathcal{E}(0)=\lambda^{2}$, such that the solution blows up in finite time. If we replace $b|u|^{p-1} u$ by $b|u|^{p-1} u-q(x)^{2} u$, where $q(x)$ is a sufficiently slowly decreasing function, an analogous result holds.
\end{abstract}

\section{INTRODUCTION AND BACKGROUND}

Suppose that $f$ is a continuous real valued function defined on $R^{n} \times R^{1}$ and $u_{u}, v_{0}$ are "nice" functions defined on $R^{n}$ in a sense to be made precise below. Suppose also that $0<T<+\infty$.

There is extensive literature on the question of the long time existence-global nonexistence of solutions initial value problem

$$
\begin{aligned}
u_{t t}-\Delta u & =f(x, u), & (t, x) \in[0, T) \times R^{n}, \\
u(0, x) & =u_{0}(x), u_{t}(0, x)=v_{0}(x), & x \in R^{n} .
\end{aligned}
$$

(The supremum of all $T$ 's for which the solution exists on $[0, T) \times R^{n}$ is called the lifespan of the solution of (1.1). We denote this number by $T_{\max }$. We say the solution is global if $T_{\max }=+\infty$ while it is nonglobal if $T_{\max }<+\infty$. If $0<T_{\max }<$ $+\infty$ we say that the solution blows up in finite time. See 11.)

See for example 20 and the papers cited therein. Some relevant earlier papers include [3, 8]. The forcing term $f$, of primary interest is the function $f(x, u)=$ $|u|^{p-1} u-\mu^{2} u$ when $p>1$. We refer to the case $\mu=0$ as the mass free case and the case $\mu \neq 0$ as the mass case. A special case of the abstract result of [8] applied to (1.1), (1.2) states the following:

Theorem 1. Suppose that there is $\epsilon>0$ such that for all possible real values of $x, u$ we have $u f(x, u) \leq(2+\epsilon) F(x, u)$ where $F_{u}(x, u)=f(x, u)$. Suppose further

Received by the editors May 11, 1999.

1991 Mathematics Subject Classification. Primary 35L15, 35Q72.

The first author was supported in part by NATO grant CRG-95120. The second author was supported by the Institute for Theoretical and Applied Physics at Iowa State University.

(C)2000 American Mathematical Society 
that for some $T>0$ (1.1), (1.2) has a solution. Assume that the initial energy

$$
\mathcal{E}(0) \equiv \frac{1}{2} \int_{R^{n}}\left|\nabla u_{0}(x)\right|^{2} d x+\frac{1}{2} \int_{R^{n}} v_{0}^{2}(x)-\int_{R^{n}} F\left(x, u_{0}(x)\right) d x
$$

is negative. Then $T_{\max }<+\infty$. (The initial data are taken to be such that every integral in (1.3) is absolutely convergent.

The principle extension of this result relevant to this paper is due to Straughan [19]. He proved that if

$$
0 \leq \mathcal{E}(0) \leq \frac{\left(u_{0}, v_{0}\right)_{L^{2}\left(R^{n}\right)}^{2}}{\left\|u_{0}\right\|_{L^{2}\left(R^{n}\right)}^{2}}
$$

and

$$
\left(u_{0}, v_{0}\right)_{L^{2}\left(R^{n}\right)} \geq 0
$$

where $\left(u_{0}, v_{0}\right)_{L^{2}\left(R^{n}\right)}$ denotes the scalar product in $L^{2}\left(R^{n}\right)$, then the conclusion of Theorem 1 continues to hold. Some further sharpening of this result is possible in the mass case using the ideas of 17 for bounded domains. We shall describe this situation more precisely below. Like the result of [8], the result of [19] has an abstract formulation in Hilbert space.

There is of course much more to this business than we have indicated here. For example, there are many related results in bounded domains [3, 5, 17, 22, as well as for abstract problems [8, 9] 22.

However until recently there has been very little work on the question of global existence and global nonexistence for solutions of the initial value problem for semilinear wave equations which also have damping terms present. Indeed, to our knowledge, the first extensions of Theorem 1 to equations which include linear damping terms $(m=1)$ and nonlinear sources were given in [8, 9] by means of "concavity arguments". The most general such abstract result is contained in the following theorem [9]:

Suppose $H$ is a Hilbert space with inner product $(\cdot, \cdot)$, where $D_{A}, D_{A_{1}}, D_{P}$ are densely defined subspaces on which self-adjoint linear operators $P: D_{P} \rightarrow H$, $A: D_{A} \rightarrow H$ and $A_{1}: D_{A_{1}} \rightarrow H$ are given. Let $D$ be a fourth subspace of $H$ and $F$ be a gradient operator from $D$ into its dual $D^{\prime}$, i.e., there is a potential $\mathcal{F}: D \rightarrow C$ (or $R$ ) such that

$$
\mathcal{F}^{\prime}(x) \cdot y=(F(x), y)_{D},
$$

where $\mathcal{F}^{\prime}$ denotes the Fréchet derivative of $\mathcal{F}$ and $(,)_{D}$ is the natural pairing in $D$. Let $(P x, x)>0$ for $x \neq 0,(A x, x) \geq 0$ and $\left(A_{1} x, x\right) \geq 0$ in the appropriate operator domain. In [9] the following result was established:

Theorem 2. If $u(\cdot)$ is a solution of

$$
\begin{gathered}
P u_{t t}+A_{1} u_{t}+A u-F(u)=0, \\
u(0)=u_{0}, \quad u_{t}(0)=v_{0},
\end{gathered}
$$

with $u(t) \in D \cap D_{A} \cap D_{P}, u_{t}(t) \in D_{A_{1}} \cap D_{P}$ and $u_{t t}(t) \in D_{P}$ for $t \in[0, T), T \leq \infty$, and with negative initial energy $\mathcal{E}(0)$, i.e., with

$$
\mathcal{E}(0) \equiv \frac{1}{2}\left(v_{0}, P v_{0}\right)+\frac{1}{2}\left(u_{0}, A u_{0}\right)-\mathcal{F}\left(u_{0}\right)<0,
$$


then $T$ is necessarily finite provided that $F$ satisfies the following structure condition: There is a number $\alpha>0$ such that

$$
(F(x), x)_{D} \geq(4 \alpha+2) \mathcal{F}(x)
$$

for all $x \in D$.

Specifically, instead of (1.1), (1.2), we have in mind initial value problems of the form

$$
\begin{aligned}
u_{t t}+Q\left(t, x, u_{t}\right)-\Delta u & =f(x, u), & (t, x) \in[0, \infty) \times R^{n}, \\
u(0, x)=u_{0}(x), \quad u_{t}(0, x) & =v_{0}(x), & x \in R^{n} .
\end{aligned}
$$

Here it is assumed that $v Q(t, x, v) \geq 0, Q(t, x, 0)=f(0)=0$ and $f(x, u) \sim u|u|^{p-2}$ for large $|u|$.

However, the interaction between the nonlinear damping and source terms creates difficulties which were overcome, for a bounded domain with Dirichlet boundary conditions, by Georgiev and Todorova [2]. The abstract version was studied by Levine and Serrin [13] and by Levine, Pucci and Serrin 12. Thereafter, in a series of papers the same technique was used in studying the behaviour of solutions of related equations (see Ono [16]).

Indeed, several authors (see Ikehata [4, Ohta [15], Vitillaro [27]) applied the Payne and Sattinger potential well arguments [17 for [1.8)-(1.9) in the bounded domain case.

In this paper we shall consider the following simple prototype of (1.8)-(1.9), namely

$$
\begin{array}{rr}
u_{t t}+a\left|u_{t}\right|^{m-1} u_{t}-\Delta u=f(x, u), & (t, x) \in[0, \infty) \times R^{n}, \\
u(0, x)=u_{0}(x), \quad u_{t}(0, x)=v_{0}(x), & x \in R^{n},
\end{array}
$$

where $f(x, u)=|u|^{p-1} u-q(x)^{2} u$ and $q(x)$ is a locally bounded measurable function on $R^{n}$.

Insofar as local existence is concerned, we have for (1.10)-1.11):

Theorem 3 (Local existence). Suppose that $1 \leq p<n /(n-2)$ if $n>2$ or $1 \leq p$ if $n=1,2$. Then, for any compactly supported data

$$
u_{0} \in W^{1,2}\left(R^{n}\right), \quad v_{0} \in L^{2}\left(R^{n}\right),
$$

the Cauchy problem (1.10)-(1.11) has a unique solution such that

$$
\begin{gathered}
u(t, x) \in C\left([0, T) ; W^{1,2}\left(R^{n}\right)\right), \\
u_{t}(t, x) \in C\left([0, T) ; L^{2}\left(R^{n}\right)\right) \cap L^{m+1}\left([0, T) \times R^{n}\right)
\end{gathered}
$$

for sufficiently small $T>0$.

This result follows from the corresponding theorem in bounded domains [2] in view of the finite speed of propagation principle.

More recently and independently, in [11, 23, 24] the following result was established for the Cauchy problem (1.10)-(1.11):

Theorem 4. Assume that $u_{0}, v_{0}$ belong to $H^{1}\left(R^{n}\right)$ and $L^{2}\left(R^{n}\right)$ and have compact support. Let $f(x, u)=b|u|^{p-1} u-\mu^{2} u, \mu^{2} \geq 0$.

(a) If $1 \leq m<p$, then every local solution is global. (This is also contained in [21].) 
(b) If $\mu=0, \mathcal{E}(0)<0$ and $p>m>n p /(n+p+1)$, then the local solution blows up in finite time.

(c) If $\mu=0, \mathcal{E}(0)<<0,\left(u_{0}, v_{0}\right)_{L^{2}\left(R^{n}\right)} \geq 0$ and $1 \leq m \leq n p /(n+p+1)$, then the local solution blows up in finite time.

(d) If $\mu^{2}>0, \mathcal{E}(0)<0$ and $p>m>1$, then the local solution blows up in finite time.

Remark 1. As a matter of fact, in 23, 24] when $\mu \neq 0$, the following result was proved: If $\mu^{2}=q^{2}(x)$ is a locally bounded, measurable function on $R^{n}$ which satisfies

$$
q(x) \geq s(1+|x|)^{-\eta},
$$

where $s>0$ and

$$
0 \leq \eta \leq \frac{(p-1) m}{2(p-m)},
$$

then the conclusion of Theorem团(d) holds, i.e. the solution blows up in finite time for all negative initial energy.

This result shows that both nonlinear damping and the stabilisation term $\mu^{2} u$ are insufficient to prevent the blow up effect of the source for all negative initial energy $\mathcal{E}(0)$ in the Cauchy problem (1.10)-(1.11). Therefore, this result and the blow-up result of Levine in the case of linear damping [8] indirectly show that no condition other than $1 \leq m<p$ should be needed to guarantee the blow-up of the solution in Theorem 4 (c), i.e. the restrictions for the initial energy $\mathcal{E}(0)$ and $m$ in Theorem 4(c) are method driven. Nevertheless, since there is currently no direct proof of this we do not remove the restrictions in Theorem 4(c).

Therefore, to insure that the solution in Theorem 4 (c) blows up when $m<$ $n p /(n+p+1)$, the initial energy $\mathcal{E}(0)$ should be sufficiently negative. More precisely, there is some critical number $-\mathcal{E}^{*}<0$ depending only on $n, m, p, L$, where $L$ is a support radius for the initial data $u_{0}, v_{0}$, such that when $\mathcal{E}(0)<-\mathcal{E}^{*}$ the solution blows up in finite time. For the other cases, (b) and (d) in Theorem 4 , however, we have $\mathcal{E}^{*}=0$.

In order to deal efficiently with both cases (b), (c) simultaneously, let us define the following quantity to be employed in the sequel

$$
\mathcal{E}_{c r}= \begin{cases}0 & \text { if } m>n p /(n+p+1), \\ -\mathcal{E}^{*} & \text { if } m \leq n p /(n+p+1) .\end{cases}
$$

Next, we turn our attention to the case of positive initial energies, which, as remarked above, is the principle focus of this paper.

For the case of linear damping $(m=1)$ global nonexistence for the abstract evolution equations with positive initial energy was treated by Pucci and Serrin [18.

The following results are to be found in [25], [26]. These results are for the mass case $(\mu \neq 0)$. To describe them, we can, without loss, take $\mu=1$.

Following the ideas of the "potential well" theory introduced by Payne and Sattinger [17] (for $\mu=0$ ), we observe that in $\mathbf{R}^{n}$ the so-called depth $d$ of the potential well becomes zero (see Nakao and Ono [14]). One of the possible ways to overcome this difficulty is as follows. 
We define the functional

$$
\mathcal{J}(\phi)=\frac{1}{2}\|\nabla \phi\|_{2}^{2}+\frac{1}{2}\|\phi\|_{2}^{2}-\frac{1}{p+1}\|\phi\|_{p+1}^{p+1}
$$

for $\phi \in W^{1,2}\left(R^{n}\right)$. In this case the "potential depth", given by

$$
d=\inf \left\{\sup _{\lambda \in R} J(\lambda \phi) ; \phi \in W^{1,2}\left(R^{n}\right) \backslash\{0\}\right\},
$$

becomes positive [25]. Then for the Cauchy problem (1.10)-(1.11) with $q(x)=1$, we are able to define stable and unstable sets.

Denote

$$
W^{i} \equiv\left\{\phi \in W^{1,2}\left(R^{n}\right) \mid \mathcal{K}(\phi)>0, \quad \mathcal{J}(\phi)<d\right\} \cup\{0\}
$$

and

$$
W^{e} \equiv\left\{\phi \in W^{1,2}\left(R^{n}\right) \mid \mathcal{K}(\phi)<0, \quad \mathcal{J}(\phi)<d\right\}
$$

where

$$
\mathcal{K}(\phi)=\|\nabla \phi\|_{2}^{2}+\|\phi\|_{2}^{2}-\|\phi\|_{p+1}^{p+1}
$$

The following theorem holds [26]:

Theorem 5. Assume the hypotheses of the local existence Theorem 3 are in force and let $u(t, x)$ be a local solution of the Cauchy problem (1.10)-(1.11) on $\left[0, T_{\max }\right)$, with $f(x, u)=b|u|^{p-1} u-u$ an $1<m<p$. Then,

(a) If there exists a positive number $t_{0} \in\left[0, T_{\max }\right)$ such that $u\left(t_{0},.\right) \in W^{i}$, and $\mathcal{E}\left(t_{0}\right)<d$, the solution is global in time and $u(t,.) \in W^{i}$ for $t \geq t_{0}$.

(b) If, in addition to the assumptions in (a) we have $1<m<\min \left\{p, \frac{2}{n}+1\right\}$. Then the global solution of the Cauchy problem (1.10) -(1.11) satisfies the following decay estimate:

$$
\mathcal{E}(t) \leq C(1+t)^{-\frac{2-n(m-1)}{m-1}} .
$$

(c) If there exists a number $t_{0} \in\left[0, T_{\max }\right)$ such that $u\left(t_{0}, \cdot\right) \in W^{e}$, and $\mathcal{E}\left(t_{0}\right)<d$, then $u(t, \cdot) \in W^{e}$ for $t_{0} \in\left[0, T_{\max }\right)$ and the solution blows up in finite time, i.e. $T_{\max }<+\infty$.

The mass free case is more difficult to treat since the potential depth $d$ is zero. In this paper we develop a method to treat the mass free case. Utilizing this we are able to show that for arbitrarily large values of the initial energy, there are choices of initial data $u_{0}, v_{0}$ for which the solution blows up in finite time. In the mass case, this method allows us to consider even the more complicated problem involving a decaying mass $q(x)^{2} u$, where $q(x)$ satisfies the conditions given in Remark 1

In particular, when $q(x)=\mu>0$, we obtain an unstable set for the data in the mass case. A natural question arises: What is the relation between this unstable set and the unstable set which was found by the potential well method in [25, [26]? In fact, the two unstable sets are equivalent, in the sense that all solutions which begin, in zero time, from data belonging in either set, enter the unstable set

$$
W^{e} \equiv\left\{\phi \in W^{1,2}\left(R^{n}\right) \mid \mathcal{K}(\phi)<0, \quad \mathcal{J}(\phi)<d\right\}
$$

in finite time. 


\section{THE BLOW UP THEOREMS}

We are now in a position to state the main theorems of this paper:

Theorem 6. Consider the initial value problem

$$
\begin{array}{rr}
u_{t t}+a\left|u_{t}\right|^{m-1} u_{t}-\Delta u=b|u|^{p-1} u-q(x)^{2} u, & (t, x) \in[0, \infty) \times R^{n}, \\
u(0, x)=u_{0}(x), \quad u_{t}(0, x)=v_{0}(x), & x \in R^{n},
\end{array}
$$

with $a, b>0$. Let $\lambda>0$ be given, $1 \leq m<p$, where $p$ is as in Theorem 3 . Then there exist $u_{0} \in W^{1,2}\left(R^{n}\right)$ and $v_{0} \in L_{2}\left(R^{n}\right)$ with compact support, such that $\mathcal{E}(0)=\lambda^{2}$ and

(a) If $q(x) \neq 0$ and the conditions (1.13)-(1.14) for the decay rate of $q(x)$ are fulfilled, then the solution of the Cauchy problem (2.1)-(2.2) is not global.

(b) If $q(x)=0$ and either $m=1$ or $1<m<p$ and $m>p-2$, the solution of (2.1) -2.2 is not global.

Remark 2. It is easy to see that the lower bound $m>p-2$ in part (b) contains no further restriction on $m$ except when $n=1,2$, because, for $n \geq 3$, one has $p \leq n /(n-2) \leq 3$.

The idea of the proof is the following: We shall show under the stated hypotheses that there is initial data with prescribed positive initial energy $\mathcal{E}(0)$ such that the energy $\mathcal{E}(t)$ becomes sufficiently negative in finite time. Then we apply Theorem 4

The case $m=1$ can be established independently by means of an extension of Theorem 2] namely

Theorem 7. Let the hypotheses on $A, A_{1}, P, F(\cdot)$ of Theorem 2 be in force. Suppose the following additional condition on the nonlinearity holds:

[R] There exists $u_{0} \in D \cap D_{A} \cap D_{P}, u_{0} \neq 0$, with the property that $\mathcal{J}\left(\sigma u_{0}\right)<0$ for $\sigma>0$ and $\liminf _{\sigma \rightarrow+\infty} \sigma^{-\alpha-2}\left|\mathcal{J}\left(\sigma u_{0}\right)\right|>0$ where

$$
\mathcal{J}\left(u_{0}\right)=\frac{1}{2}\left(u_{0}, A_{0}\right)-\mathcal{F}\left(u_{0}\right) .
$$

Let $\lambda^{2}>0$ be given. Then there exist $u_{0} \in D \cap D_{A} \cap D_{P}$ and $v_{0} \in D_{A_{1}} \cap D_{P}$ such that if $u(\cdot)$ is a solution of (1.5) with $u(t) \in D \cap D_{A} \cap D_{P}, u_{t}(t) \in D_{A_{1}} \cap D_{P}$ and $u_{t t}(t) \in D_{P}$ for $t \in[0, T)$ and $\mathcal{E}(0)=\lambda^{2}$, then $T_{\text {max }}$ is necessarily finite.

\section{Proofs}

We shall first prove Theorem 7 Then we shall prove Theorem 6 with $q(x)=0$. The proof for $q(x) \neq 0$ follows from an easy modification of the proof when $q(x)=0$, as we shall see.

Proof of Theorem 7. We extend an argument of Straughan, [19], which in turn was an extension of the concavity method as applied in [9] to the functional

$$
s(t) \equiv(u(t), P u(t))+\int_{0}^{t}\left(u(\tau), A_{1} u(\tau)\right) d \tau+\left(T_{0}-t\right)\left(u_{0}, A_{1} u_{0}\right),
$$

where $T_{0}>0$ is to be chosen later. Let us assume that $u(\cdot)$ is global and that $t \leq T_{0}$. It is not hard to show that

$$
s^{\prime \prime}(t) s(t)-(\alpha+1)\left(s^{\prime}(t)\right)^{2} \geq-2(2 \alpha+1) \lambda^{2} s(t)
$$


where $\lambda^{2} \equiv \mathcal{E}(0)>0$ is given in (1.6) (see 9]). If we assume that $\left(u_{0}, P v_{0}\right)>0$, then $s^{\prime}(t)>0$ on some small interval $[0, \tau)$, say. After multiplying both sides of (3.2) by $\alpha s^{-2 \alpha-1}(t) s^{\prime}(t)$ and a quadrature we find that on $[0, \tau)$

$$
\left(s^{\prime}(t)\right)^{2} \geq 8 \lambda^{2} s(t)+\left[\left(s^{\prime}(0)\right)^{2}-8 \lambda^{2} s(0)\right]\left(\frac{s(t)}{s(0)}\right)^{2 \alpha+2} .
$$

If the quantity in brackets on the right is positive, then $s^{\prime}(t)$ cannot change sign and hence (3.3) holds on $J \equiv\left[0, T_{0}\right)$. Therefore

$$
I\left(T_{0}\right) \equiv \int_{s(0)}^{\infty}\left[8 \lambda^{2} s+\left[\left(s^{\prime}(0)\right)^{2}-8 \lambda^{2} s(0)\right]\left(\frac{s}{s(0)}\right)^{2 \alpha+2}\right]^{-1 / 2} d s \geq t
$$

on $J$. We will be done if we can find $T_{0}$ such that $T_{0}>I\left(T_{0}\right)$, since then $T_{0} \geq$ $I\left(T_{0}\right) \geq T_{\max } \geq t$. The integral on the left is impossible to evaluate. However, it can be appropriately estimated from above, since

$$
I\left(T_{0}\right) \leq\left[\left(s^{\prime}(0)\right)^{2}-8 \lambda^{2} s(0)\right]^{-1 / 2} \int_{s(0)}^{\infty}\left(\frac{s}{s(0)}\right)^{-\alpha-1} d s .
$$

We will be done if

$$
\left[\left(s^{\prime}(0)\right)^{2}-8 \lambda^{2} s(0)\right]^{-1 / 2} \frac{s(0)}{\alpha} \leq T_{0} .
$$

That is to say, we will be done if we can find $u_{0}, v_{0}$ such that

$$
\begin{aligned}
\left(u_{0}, P v_{0}\right) & >\sqrt{2} \lambda\left[\left(u_{0}, P u_{0}\right)+T_{0}\left(u_{0}, A_{1} u_{0}\right)\right]^{1 / 2}, \\
\left(u_{0}, P u_{0}\right)^{2} & \leq 4 \alpha^{2} T_{0}^{2}\left\{\left(u_{0}, P v_{0}\right)^{2}-2 \lambda^{2}\left[\left(u_{0}, P u_{0}\right)+T_{0}\left(u_{0}, A_{1} u_{0}\right)\right]\right\} .
\end{aligned}
$$

Clearly if $\lambda^{2}=0$ it is easy to find initial data such that (3.5) and (3.6) both hold.

To show that it is possible to satisfy both (3.5) and (3.6) when $\lambda^{2}>0$, we assume that $\mathcal{J}\left(u_{0}\right)<0$ and set $v_{0}=\sigma u_{0}$ where $\sigma>0$ and where

$$
\sigma^{2}=2\left(\lambda^{2}-\mathcal{J}\left(u_{0}\right)\right) /\left(u_{0}, P u_{0}\right) .
$$

Then conditions (3.5) and (3.6) become respectively

$$
\begin{aligned}
0 & >\left(u_{0}, A_{1} u_{0}\right) T_{0}+2\left(u_{0}, P u_{0}\right) \mathcal{J}\left(u_{0}\right), \\
\left(u_{0}, P u_{0}\right)^{2} & \leq 4 \alpha^{2} T_{0}^{2}\left[-2\left(u_{0}, P u_{0}\right) \mathcal{J}\left(u_{0}\right)-\left(u_{0}, A_{1} u_{0}\right) T_{0}\right] .
\end{aligned}
$$

If $\left(u_{0}, A_{1} u_{0}\right)=0$, both of these hold when $T_{0}$ is large enough. If $\left(u_{0}, A_{1} u_{0}\right)>$ 0 , then the cubic on the right of (3.8) will be maximized when $\mathcal{J}\left(u_{0}\right)<0$ and $T_{0}=-4 \mathcal{J}\left(u_{0}\right)\left(u_{0}, P u_{0}\right) / 3\left(u_{0}, A_{1} u_{0}\right)$. For this value of $T_{0}$ the equation (3.7) holds automatically, while the second inequality will hold in this (optimal) case if and only if

$$
\frac{1}{2}\left(\frac{3}{4}\right)^{3} \leq \alpha^{2} \frac{\left|\mathcal{J}\left(u_{0}\right)\right|^{3}\left(u_{0}, P u_{0}\right)}{\left(u_{0}, A_{1} u_{0}\right)^{2}} .
$$

Then by replacing $u_{0}$ by $\theta u_{0}$, letting $\theta$ become large and using condition [R], we see that we can find $u_{0}$ such that this last condition and hence (3.5) and (3.6) hold for prescribed $\mathcal{E}(0) \geq 0$.

Proof of Theorem [6] the case $q(x)=0$. We will take $a=b=1$ for convenience. Our argument is unaffected by this restriction as the reader will easily see. Suppose 
to the contrary that for given $\mathcal{E}(0)>0, u(\cdot)$ is a global solution of (2.1)-(2.2). Then, from energy identity we have

$$
\mathcal{E}(0)-\mathcal{E}(t)=\int_{0}^{t}\left\|u_{\eta}\right\|_{m+1}^{m+1} d \eta
$$

for all $t \in[0+\infty)$, where $\mathcal{E}(t) \equiv \frac{1}{2}\|\nabla u\|_{2}^{2}+\frac{1}{2}\left\|u_{t}\right\|_{2}^{2}-\frac{1}{p+1}\|u\|_{p+1}^{p+1}$.

It suffices to find $\bar{t}$ such that

$$
\int_{0}^{\bar{t}}\left\|u_{\eta}\right\|_{m+1}^{m+1} d \eta>\mathcal{E}(0)-\mathcal{E}_{c r}
$$

Then from the energy identity we have $\mathcal{E}_{c r}>\mathcal{E}(\bar{t})$ and we can apply Theorem 4 to obtain the nonexistence result.

We decompose the solution $u$ into linear and nonlinear terms as follows:

$$
u=w+v,
$$

where

$$
\begin{aligned}
0 & =w_{t t}-\Delta w, & & (t, x) \in[0, \infty) \times R^{n}, \\
w(0, x) & =u_{0}(x), \quad w_{t}(0, x)=v_{0}(x), & & x \in R^{n}
\end{aligned}
$$

and

$$
v_{t t}-\Delta v=b|u|^{p-1} u-q(x)^{2} u-a\left|u_{t}\right|^{m-1} u_{t} \equiv F(x, t), \quad(t, x) \in[0, \infty) \times R^{n},
$$

$$
v(0, x)=0, \quad v_{t}(0, x)=0, \quad x \in R^{n} .
$$

The idea of the proof is to show, as remarked above, that for appropriate data the total energy falls below $\mathcal{E}_{c r}$ in finite time. We use negative Sobolev norms to accomplish this. First, we will need a good lower bound on $\left\|u_{t}\right\|_{m+1}^{m+1}$. To do this, we first observe from Hölder's inequality and the finite speed of propagation property of solutions of (2.2) that

$$
\left\|u_{t}\right\|_{2} \leq C(t+L)^{n / q}\left\|u_{t}\right\|_{m+1},
$$

where $1 / q+1 /(m+1)=1 / 2$. Additionally, we need the inequality

$$
\left\|u_{t}\right\|_{2} \geq C\left\|u_{t}\right\|_{H^{-s}\left(R^{n}\right)} \equiv C\left\|u_{t}\right\|_{H^{-s}}
$$

for some $C=C(s, n)$, which is valid if $s / n \geq 0$. Combining these with the decomposition of the solution (3.9), we have

$$
\left[(t+L)^{n / q}\left\|u_{t}\right\|_{m+1}\right]^{m+1} \geq\left\|u_{t}\right\|_{2}^{m+1} \geq C\left[\left\|w_{t}\right\|_{H^{-s}}^{m+1}-\left\|v_{t}\right\|_{H^{-s}}^{m+1}\right]
$$

where $C$ is another computable constant. This gives us the desired lower bound for $\left\|u_{t}\right\|_{m+1}^{m+1}$ on any interval $[0, T)$ of fixed, finite length, $T \leq L$ say, provided we can find good estimates for the terms on the extreme right-hand side of (3.14). We now proceed to estimate these terms.

We obtain, in turn,

1. an estimate for $\left\|w_{t}\right\|_{H^{-s}}^{m+1}$ from below.

2. an estimate for $\left\|v_{t}\right\|_{H^{-s}}^{m+1}$ from above. This estimate is based on the next estimate 3 .

3. an estimate for $E(t) \equiv\|\nabla u\|_{2}^{2}+\left\|u_{t}\right\|_{2}^{2}$ from above. 
These estimates hold for a small time interval. Using them, we are able to estimate the rate of decrease of the energy $\mathcal{E}(t)$ from below in this time interval. Then, we show that for any initial energy $\mathcal{E}(0)$ we can choose $v_{0}, u_{0}$ with this initial energy such that for some $t_{0}$ in the above time interval, the energy $\mathcal{E}\left(t_{0}\right)<\mathcal{E}_{c r}$. Then an application of Theorem 4 for blow-up of solutions for energies $\mathcal{E}\left(t_{0}\right)<\mathcal{E}_{c r}$ enables us to complete the proof.

We begin with the linear term $w$. In terms of the Fourier transform variable $\xi$, we can write the solution of the free equation (3.10)-3.11) as follows:

$$
\hat{w}(\xi, t)=\cos (t|\xi|) \hat{u}_{0}(\xi)+\frac{\sin (t|\xi|)}{|\xi|} \hat{v}_{0}(\xi) .
$$

Computing $\hat{w}_{t}$, squaring, and using the inequalities $(a+b)^{2} \geq a^{2} / 2-b^{2}, \sin ^{2} \alpha \leq \alpha^{2}$ and $\cos ^{2} \alpha \geq 1-2 \alpha^{2}$, we find that

$$
\left|\hat{w}_{t}\right|^{2}(t, \xi) \geq \frac{1}{2}\left|\hat{v}_{0}(\xi)\right|^{2}-t^{2}|\xi|^{2}\left[|\xi|^{2}\left|\hat{u}_{0}(\xi)\right|^{2}+\left|\hat{v}_{0}(\xi)\right|^{2}\right] .
$$

We have after multiplying by $\left(1+|\xi|^{2}\right)^{-s}$ for $s>2$, and integrating over $\xi \in R^{n}$

$$
\left\|w_{t}\right\|_{H^{-s}}^{2}+S(L, n) t^{2} E(0) \geq \frac{1}{2}\left\|v_{0}\right\|_{H^{-s}}^{2}
$$

for some appropriate $S(L, n)>1$. Raising both sides of this inequality to the $(m+1) / 2$ power, we find for some suitable $C>0$,

$$
\left\|w_{t}\right\|_{H^{-s}}^{m+1} \geq C\left[\left\|v_{0}\right\|_{H^{-s}}^{m+1}-t^{m+1} E^{(m+1) / 2}(0)\right] .
$$

We turn next to the second term. Taking the $H^{-s}$ scalar product of both sides of (3.12) with $v_{t}$ it follows in the usual manner that there is a constant $C$ such that

$$
\left\|v_{t}\right\|_{H^{-s}} \leq C \int_{0}^{t}\|F(\cdot, \eta)\|_{H^{-s}} d \eta
$$

where $F$ is defined in (3.12). We have that $\|g\|_{H^{-s}} \leq C\|g\|_{r}$ as long as $r \leq 2$ and $s \geq n(1 / r-1 / 2)$. We use this estimate twice, once with $g=u|u|^{p-1}$ and $r=2$ and once with $g=u_{t}\left|u_{t}\right|^{m-1}$ and $r=(m+1) / m$, in the inequality

$$
\|F(\cdot, t)\|_{H^{-s}} \leq\left\|u|u|^{p-1}\right\|_{H^{-s}}+\left\|u_{t}\left|u_{t}\right|^{m-1}\right\|_{H^{-s}} .
$$

Now we choose $s>\max \left\{2, \frac{n(m-1)}{2(m+1)}\right\}$. We obtain

$$
\|F(\cdot, t)\|_{H^{-s}} \leq C\left(\|u\|_{2 p}^{p}+\left\|u_{t}\right\|_{m+1}^{m}\right) \leq C\left[(L+t)^{p_{n}}\|\nabla u\|_{2}^{p}+\left\|u_{t}\right\|_{m+1}^{m}\right],
$$

with $p_{n} \equiv(n-(n-2) p) / 2 p$, which is strictly positive for $n>2$ in view of our restrictions on $p$. In the above estimate we used the finite propagation speed property of the solution and the Hölder and Poincaré inequalities after noting that $2 p \leq 2 n /(n-2)$ for $n>2$.

After integrating both sides of (3.21) and using the Hölder inequality with respect to $t$ in the integral $\int_{0}^{t}\left\|u_{\eta}\right\|_{m+1}^{m} d \eta$, we obtain,

$$
\begin{aligned}
\int_{0}^{t} \| & F(\cdot, \eta) \|_{H^{-s}} d \eta \\
& \leq\left[(L+t)^{p_{n}} \int_{0}^{t} E^{p / 2}(\eta) d \eta+t^{1 /(m+1)}\left(\int_{0}^{t}\left\|u_{\eta}\right\|_{m+1}^{m+1} d \eta\right)^{m /(m+1)}\right] .
\end{aligned}
$$


Notice that for our problem, $E(t) \geq \mathcal{E}(t)$. Since we are assuming that $\mathcal{E}(t) \geq \mathcal{E}_{c r}$, we have

$$
\int_{0}^{t}\left\|u_{\eta}\right\|_{m+1}^{m+1} d \eta=\mathcal{E}(0)-\mathcal{E}(t) \leq E(0)-\mathcal{E}_{c r}
$$

and consequently (as long as $t \leq L$, say)

$$
\int_{0}^{t}\|F(\cdot, \eta)\|_{H^{-s}} d \eta \leq C(L)\left[\int_{0}^{t} E^{p / 2}(\eta) d \eta+t^{1 /(m+1)}\left(E(0)-\mathcal{E}_{c r}\right)^{m /(m+1)}\right] .
$$

In order to complete our estimate for $\left\|v_{t}\right\|_{H^{-s}}$, we will need an upper bound for $E(t)$ which we obtain as follows: We have

$$
\begin{aligned}
E^{\prime}(t) & \leq\left(u_{t}, u|u|^{p-1}\right) \quad \leq C\left\|u_{t}\right\|_{2}\|u\|_{2 p}^{p} \\
& \leq C(L+t)^{p_{n}}\left\|u_{t}\right\|_{2}\|\nabla u\|_{2}^{p} \leq C(L+t)^{p_{n}} E^{(p+1) / 2}(t),
\end{aligned}
$$

where the constant $C$ changes as we move from left to right.

Let $t<L$. From (3.23) and a quadrature, we obtain

$$
E(t) \leq \frac{E(0)}{\left(1-C E(0)^{(p-1) / 2} t\right)^{2 /(p-1)}} .
$$

We see that there exists $T_{1}=C(p, n, L) E^{-(p-1) / 2}(0)$ such that as long as $t<T_{m}=$ $\min \left\{L, T_{1}\right\}$ we have $E(t) \leq 2 E(0)$.

Combining these observations with (3.19) and (3.22), we find that for $t \in\left[0, T_{m}\right)$

$$
\left\|v_{t}\right\|_{H^{-s}}^{m+1} \leq C(m, n, p, L)\left[t^{m+1} E^{p(m+1) / 2}(0)+t\left[E(0)-\mathcal{E}_{c r}\right]^{m}\right] .
$$

Let $C_{1}=\max (1, C(m, n, p, L))$. Using (3.18) and (3.24) we have, for some computable constant $C$ and $t \in\left[0, T_{m}\right)$

$$
\begin{aligned}
\left\|u_{t}\right\|_{m+1}^{m+1} \geq C\left[\left\|v_{0}\right\|_{H^{-s}}^{m+1}-C_{1} t^{m+1} E^{p(m+1) / 2}(0)\right. & \\
& \left.-t^{m+1} E^{(m+1) / 2}(0)-C_{1} t\left[E(0)-\mathcal{E}_{c r}\right]^{m}\right] .
\end{aligned}
$$

From now on, we require that $E(0)>1$. Then we have $C_{1} t^{m+1} E^{p(m+1) / 2}(0) \geq$ $t^{m+1} E^{(m+1) / 2}(0)$ and the above inequality may be weakened to

$$
\left\|u_{t}\right\|_{m+1}^{m+1} \geq C\left[\left\|v_{0}\right\|_{H^{-s}}^{m+1}-2 C_{1} t^{m+1} E^{p(m+1) / 2}(0)-C_{1} t\left[E(0)-\mathcal{E}_{c r}\right]^{m}\right],
$$

which holds for $t \in\left[0, T_{m}\right)$.

Integrating both sides of this inequality we obtain

$$
\int_{0}^{t}\left\|u_{\eta}\right\|_{m+1}^{m+1} d \eta \geq C t\left[\left\|v_{0}\right\|_{H^{-s}}^{m+1}-\left(\frac{2 C_{1}}{m+2}\right) t^{m+1} E^{p(m+1) / 2}(0)-\frac{C_{1}}{2} t\left[E(0)-\mathcal{E}_{c r}\right]^{m}\right]
$$

for $t \in\left[0, T_{m}\right)$. If we can find $t \in\left[0, T_{m}\right)$ and $t>\left(E(0)-\mathcal{E}_{c r}\right) E^{-p(m+1) / 2 m}(0)$, then

$$
t^{m+1} E^{p(m+1) / 2}(0) \geq t\left[E(0)-\mathcal{E}_{c r}\right]^{m} .
$$


Consequently for such values of $t$ we have, from (3.27),

$$
\int_{0}^{t}\left\|u_{\eta}\right\|_{m+1}^{m+1} d \eta \geq C t\left[\left\|v_{0}\right\|_{H^{-s}}^{m+1}-\left(\frac{(m+6) C_{1}}{2(m+2)}\right) t^{m+1} E^{p(m+1) / 2}(0)\right] .
$$

We set $t_{1}=\left\|v_{0}\right\|_{H^{-s}} E^{-p / 2-\delta}(0)$, where $0<\delta<\min \left(\frac{p-m}{2 m}, \frac{m+2-p}{2}\right)$. By hypothesis $p>m$ and $m+2>p$. We will show that $T_{m}>t_{1}>\left(E(0)-\mathcal{E}_{c r}\right) E^{-p(m+1) / 2 m}(0)$.

We set $v_{0}=\rho \phi$ and $u_{0}=\sigma \psi$ where $\rho, \sigma>0$ and are such that $\mathcal{E}(0)=\lambda^{2}$ is independent of these variables (this implies that $\sigma^{p+1} / \rho^{2}=o(1)$ as $\rho \rightarrow+\infty$ ). Clearly, as $\rho \rightarrow+\infty, E(0) \rightarrow+\infty$ as well.

Since $\left\|v_{0}\right\|_{H^{-s}} \leq\left\|v_{0}\right\|_{2} \leq \sqrt{E(0)}$, the inequality $t_{1}<T_{m}=\min \left\{L, T_{1}\right\}$ will hold provided $E^{-(p-1) / 2-\delta}(0)<C E^{-(p-1) / 2}(0)$ and $E^{-(p-1) / 2-\delta}(0)<L$. The later inequalities clearly hold for $\rho$ so large that $E(0)>\max \left((1 / C)^{1 / \delta},(1 / L)^{\frac{1}{(p-1) / 2+\delta}}\right)$.

To show that $t_{1}>\left(E(0)-\mathcal{E}_{c r}\right) E^{-p(m+1) / 2 m}(0)$, we define $\epsilon \equiv\|\phi\|_{H^{-s}} /\|\phi\|_{2}$. Then $t_{1}>\left(E(0)-\mathcal{E}_{c r}\right) E^{-p(m+1) / 2 m}(0)$, provided

$$
\left\|v_{0}\right\|_{H^{-s}} E^{-\delta}(0)>E^{-p / 2 m}(0)\left[E(0)-\mathcal{E}_{c r}\right] .
$$

The above inequality holds if

$$
\epsilon\left\|v_{0}\right\|_{2} E^{-\delta}(0)>E^{1-p / 2 m}(0)\left[1-\mathcal{E}_{c r} / E(0)\right] .
$$

This last inequality will hold for our choice of initial data and for all sufficiently large $\rho$ because as $\rho \rightarrow+\infty$, the left-hand side grows like $\rho^{1-2 \delta}$ while the right-hand side grows like $\rho^{2-p / m}$ while $1-2 \delta>2-p / m$ in view of our choice of $\delta$.

Setting $t=t_{1}$ in (3.28) we will be done if

$$
\begin{aligned}
\int_{0}^{t_{1}}\left\|u_{\eta}\right\|_{m+1}^{m+1} d \eta & \geq C\left\|v_{0}\right\|_{H^{-s}}^{m+2} E^{-p / 2-\delta}(0)\left[1-\left(\frac{(m+6) C_{1}}{2(m+2)}\right) E^{-\delta(m+1)}(0)\right] \\
& \geq \mathcal{E}(0)-\mathcal{E}_{c r}
\end{aligned}
$$

For sufficiently large $\rho$ we have $\frac{(m+6) C_{1}}{2(m+2)} E^{-\delta(m+1)}(0)<1 / 2$. Thus, the inequality (3.29) will hold provided

$$
\int_{0}^{t_{1}}\left\|u_{\eta}\right\|_{m+1}^{m+1} d \eta \geq C / 2 \epsilon^{m+2}\left\|v_{0}\right\|_{2}^{m+2} E^{-\delta-p / 2}(0)>\mathcal{E}(0)-\mathcal{E}_{c r}
$$

and this inequality will in turn hold for sufficiently large $\rho$ provided $m+2>p+2 \delta$. The latter condition is fulfilled due to the choice of $\delta$.

Finally, when $m<n p /(n+p+1)$ we can guarantee the last blow-up condition from Theorem 4 (c), namely $\left(u_{0}, v_{0}\right)_{L^{2}\left(R^{n}\right)} \geq 0$, by replacing (if necessary) $v_{0}(x)$ with $\operatorname{sign}\left(u_{0}, v_{0}\right) v_{0}(x)$.

This, together with Theorem 4, completes the proof when $q(x)=0$.

Proof of Theorem 6 , the case $q(x) \neq 0$. The proof follows the same lines as before with slight modifications. Using (3.12), the estimates (3.20) and (3.21) must be written as follows:

$$
\|F(\cdot, t)\|_{H^{-s}} \leq\left\|u|u|^{p-1}\right\|_{H^{-s}}+\left\|u_{t}\left|u_{t}\right|^{m-1}\right\|_{H^{-s}}+\|q u\|_{H^{-s}}
$$

and

$$
\begin{aligned}
\|F(\cdot, t)\|_{H^{-s}} & \leq C\left(\|u\|_{2 p}^{p}+\left\|u_{t}\right\|_{m+1}^{m}+\|q u\|_{2}\right) \\
& \leq C\left[(L+t)^{p_{n}}\|\nabla u\|_{2}^{p}+\left\|u_{t}\right\|_{m+1}^{m}+\|q u\|_{2}\right] .
\end{aligned}
$$


Then after a quadrature and an application of Hölder's inequality with respect to $t$ in the integral $\int_{0}^{t}\left\|u_{\eta}\right\|_{m+1}^{m} d \eta$, we find that

$$
\int_{0}^{t}\|F(\cdot, \eta)\|_{H^{-s}} d \eta \leq C(L)\left[\int_{0}^{t}\left[E^{p / 2}(\eta)+E^{1 / 2}(\eta)\right] d \eta+t^{1 /(m+1)} E^{m /(m+1)}(0)\right]
$$

where $E(t)=\|\nabla u\|_{2}^{2}+\left\|u_{t}\right\|_{2}^{2}+\|q u\|_{2}^{2}$. In the above inequality we have used the fact that if $q(x)$ satisfies the conditions (1.13)-(1.14), the solution of the Cauchy problem (2.1)-(2.2) is not global for any negative initial energy (see 23], 24]). Therefore, the constant $\mathcal{E}_{c r}$ from the mass free case is now zero. We obtain the same upper bound for $E(t)$ as before on the same time interval [0, $\left.T_{m}\right)$. We can then rewrite the estimate (3.25) as follows:

$$
\left\|u_{t}\right\|_{m+1}^{m+1} \geq C\left[\left\|v_{0}\right\|_{H^{-s}}^{m+1}-C_{1} t^{m+1} E^{p(m+1) / 2}(0)-t^{m+1} E^{(m+1) / 2}(0)-C_{1} t E(0)^{m}\right] .
$$

As before, since $E(0)>1$ and $C_{1} \geq 1$, the third term on the right-hand side of the above inequality dominates the second one. The remainder of the proof coincides with the remainder of the proof in the mass free case, but is simplified since we have now $\mathcal{E}_{c r}=0$.

\section{ACKNOWLEDGEMENT}

The authors would like to thank the referee for some suggestions that improved the readability of the manuscript.

\section{REFERENCES}

[1] BALl, J., Remarks on blow-up and nonexistence theorems for nonlinear evolution equations, Quart. J. Math. Oxford (2) 28 (1977), 473-486. MR 57:13150

[2] Georgiev, V. And G. Todorova, Existence of a solution of the wave equation with nonlinear damping and source terms, J. Diff. Eqs. 109 (1994), 295-308. MR 95b:35141

[3] Glassey, R. T., Blow-up theorems for nonlinear wave equations, Math. Z. 132 (1973), 183203. MR 49:5549

[4] Ikehata, R., Some remarks on the wave equations with nonlinear damping and source terms Nonl. Anal. T. M. A (to appear). MR 97i:35117

[5] Kawarada, K., On solutions of nonlinear wave equations, J. Phys. Soc. Jap. (1971), 280-282.

[6] Keller, J. B., On solutions of nonlinear wave equations, Comm. Pure Appl. Math 10 (1957), 523-530. MR 20:3371

[7] Knops, R. J., Levine, H. A. and Payne L. E., Nonexistence, instability and growth theorems for solutions to an abstract nonlinear equation with applications to elastodynamics, Arch. Rational Mech. Anal. 55 (1974), 52-72. MR 51:1093

[8] Levine, H.A., Instability and nonexistence of global solutions of nonlinear wave equations of the form $P u_{t t}=-A u+F(u)$, Trans. Amer. Math. Soc. 192 (1974), 1-21. MR 49:9436

[9] Levine, H.A., Some additional remarks on the nonexistence of global solutions to nonlinear wave equations, SIAM J. Math. Anal. 5 (1974), 138-146. MR 53:3525]

[10] Levine, H.A., Nonexistence of global weak solutions to some properly and improperly posed problems of mathematical physics: The method of unbounded Fourier coefficients, Math. Ann. 214 (1975), 205-220. MR 52:6200

[11] Levine, H.A., Park, S. R. And Serrin, J., Global existence and global nonexistence of solutions of the Cauchy problem for a nonlinearly damped wave equation, JMAA 228 (1998), 181-205. MR 99k:35124

[12] Levine, H.A., Pucci, P. ANd Serrin, J., Some remarks on global nonexistence for nonautonomous abstract evolution equations, Contemporary Math. 208 (1997), 253-263. MR 98j:34124 
[13] Levine, H.A. And Serrin, J., A global nonexistence theorem for quasilinear evolution equations with dissipation, Arch. Rational. Mech. Anal. 137 (1997), 341-361. MR 99b:34110

[14] NAKaO, M. AND Ono, K., Existence of global solutions to the Cauchy problem for the semilinear dissipative wave equations, Math. Z. 214 (1993), 325 - 342. MR 94h:35167

[15] Онта, M., Remarks on blow up of solutions for nonlinear evolution equations of second order, (to appear). MR 99m:35167

[16] ONo, K. On global solutions and blowup solutions of nonlinear Kirchhoff strings with nonlinear dissipation, J. Math. Anal. and Appl. 216 (1997), 321-342. MR 99e:35219

[17] Payne, L. E. and Sattinger, D., Saddle points and instability of nonlinear hyperbolic equations, Israel Math. J. 22 (1981), 273-303. MR 53:6112

[18] Pucci, P. And Serrin, J. Global nonexistence for abstract evolution equations with positive initial energy, JDE 109 (1998)). MR 2000a:34119

[19] Straughan, B., Further global nonexistence theorems for abstract nonlinear wave equations Proc. AMS 48 (2)(1975) 381-390. MR 51:1518

[20] Strauss, W., Nonlinear Wave Equations CBMS Regional Conference Series in Mathematics, Am. Math. Soc. 73(1989). MR 91g:35002

[21] Strauss, W., The Energy Method in Nonlinear Partial Differential Equations, Notas De Matematics \#47, Istit. di Mat. Conselho Nacional de Pesquisas, Rio de Janeiro 1969. MR 42:8051

[22] Tsutsumi, H., On solutions of semilinear differential equations in a Hilbert space, Math. Japonicea 17 (1972), 173-193.

[23] Todorova, G., The Cauchy problem for nonlinear wave equations with nonlinear damping and source terms J. Nonl. Anal. TMA 41 7-8 (2000), 891-905.

[24] Todorova, G., The Cauchy problem for nonlinear wave equations with nonlinear damping and source terms C. R. Acad. Sci. Paris 326 Série I (1998), 191-196. MR 99e:35154

[25] Todorova, G., Stable and unstable sets for the Cauchy problem for a nonlinear wave equation with nonlinear damping and source terms C. R. Acad. Sci. Paris 328 Série I (1999), 117122. MR 99j:35144

[26] Todorova, G., Stable and unstable sets for the Cauchy problem for a nonlinear wave equation with nonlinear damping and source terms JMAA 239 (1999), 213-226. CMP 2000:04

[27] Vitillaro, E., Global nonexistence theorems for a class of evolution equations with dissipation Arch. Rat. Mech. Anal., 149, 2(1999), 155-182. MR 2000:04

Department of Mathematics, Iowa State University, Ames, Iowa 50011

E-mail address: halevine@iastate.edu

Institute of Mathematics, Bulgarian Academy of Sciences, Boul. Acad. Bonchev BL.8, Sofia 1113, Bulgaria

E-mail address: grozdena@bas.bg

Current address: School of Mathematics, University of Minnesota, Minneapolis, Minnesota 55455

E-mail address: todorova@math.umm.edu 